\title{
Photocatalytic Activity of Nanosized Cadmium Sulfides Synthesized by Complex Compound Thermolysis
}

\author{
Yingchun Yu, Youxian Ding, Shengli Zuo, and Jianjun Liu \\ Faculty of Sciences, Beijing University of Chemical Technology, Beijing 100029, China \\ Correspondence should be addressed to Jianjun Liu, jjliu717@yahoo.com.cn
}

Received 15 June 2010; Accepted 15 August 2010

Academic Editor: Mohamed Sabry Abdel-Mottaleb

Copyright () 2011 Yingchun Yu et al. This is an open access article distributed under the Creative Commons Attribution License, which permits unrestricted use, distribution, and reproduction in any medium, provided the original work is properly cited.

The nanosized cadmium sulfides (CdSs) containing different phase structures were synthesized via the complex compound thermolysis method using different molar ratio of thiourea to acetate cadmium (S/Cd) and characterized by XRD, TEM, UVVis, and IR spectra. The results of photocatalytic degradation of rhodamine B (RB) show that the activity order of CdS concerning the phase compositions is of cubic > hexagonal > cubic + hexagonal, where the CdS with the cubic phase has the best photocatalytic degradation activity of RB due to its larger abilities of adsorption and absorbance and smaller particle size of about $10 \sim 13 \mathrm{~nm}$.

\section{Introduction}

Nanomaterials are assemblies with particles or crystal size between $1 \mathrm{~nm}$ and $100 \mathrm{~nm}$, which include different types of nanoparticles, nanowires, nanotubes, and nanofilms and other nanobulk materials. Nanomaterials possess many unique physical and chemical properties different from their bulk counterparts due to the obvious quantum size effect and small size effect $[1,2]$. Photocatalysis is one of the key technologies to solve both energy and environmental problems using solar energy, in which an efficient photocatalyst is necessary. Although remarkable progress has been made in recent years for photocatalysts working under ultraviolet light, there are limitations for the photocatalysts to be applied under visible light. The problem could be resolved by using visible light-induced photocatalysts with proper band gap energy, such as $\mathrm{CdS}, \mathrm{WO}_{3}$, or $\mathrm{Fe}_{2} \mathrm{O}_{3}$. Although $\mathrm{CdS}$ is a good candidate for photocatalytic water reduction and pollutant oxidation, it has the fatal photocorrosion problem due to the oxidation of itself by the photogenerated hole [3]. Usually, CdSs are of cubic phase and hexagonal phase and the cubic phase can transform into hexagonal one when heated to certain temperatures [4]. At present, there are many studies on the synthesis of hexagonal CdS. Lin et al. [5] and Bao et al. [6] found that the hexagonal
CdS nanoparticles synthesized via solvothermal method have much better photocatalytic activity for degradation of methylene blue and better hydrogen production activity for water photolysis, using thiourea and cadmium acetate as raw materials. Jing and Guo [7] reported a CdS catalyst system with a high hydrogen production activity for water photolysis using cadmium acetate as cadmium source and $\mathrm{H}_{2} \mathrm{~S}$ as sulfur source. The CdS synthesized under solvothermal or heating conditions is usually of stable hexagonal phase, but there are a few reports on the synthesis of metastable cubic CdS. Many factors can influence the photocatalytic activity of CdS, such as phase composition, crystallite size, morphology, specific surface area, and energy gap. For example, Degussa P25 (made in Germany), a type of commercial nanosized titania which possesses a high photocatalytic activity, exists in polymorphs composed of about $80 \%$ anatase and $20 \%$ rutile [8]. Therefore, it is very important to study how to get the CdS photocatalysts with different phase compositions and their photocatalytic activities by controlling reaction conditions.

In this study, the CdS nanocrystals with different phase compositions were synthesized via thermal decomposition of thiourea-cadmium complexes using cadmium acetate and thiourea as raw materials. The photocatalytic activities of the synthesized CdS nanocrystals for the degradation of Rhodamine B under visible light were also studied. 
TABLE 1: Synthesis conditions, phase composition, and crystalline size of CdS photocatalysts.

\begin{tabular}{|c|c|c|c|c|c|}
\hline Sample no. & $c\left(\mathrm{CS}\left(\mathrm{NH}_{2}\right)_{2}\right) /\left(\mathrm{mol} \cdot 1^{-1}\right)$ & $c\left(\mathrm{Cd}^{2+}\right) /\left(\mathrm{mol} \cdot \mathrm{l}^{-1}\right)$ & $n(\mathrm{~S}) / n(\mathrm{Cd})$ & Phase composition & Grain size/nm \\
\hline A & 0.07 & 0.14 & 0.5 & $\mathrm{CdS}($ cubic $)+\mathrm{CdO}$ & 10 \\
\hline B & 0.14 & 0.14 & 1 & Cubic & 13 \\
\hline $\mathrm{C}$ & 0.21 & 0.14 & 1.5 & Cubic + hexagonal & 17 \\
\hline $\mathrm{D}$ & 0.28 & 0.14 & 2 & Hexagonal & 26 \\
\hline $\mathrm{E}$ & 0.42 & 0.14 & 3 & Hexagonal & 26 \\
\hline
\end{tabular}

* The grain size of CdS can be calculated by using the crystal plane of cubic (111) and hexagonal (002), respectively.

\section{Experimental}

2.1. Synthesis of CdS. All chemicals were of analytical grade and used without further purification. The preparation conditions and analysis results of CdS samples synthesized using different S/Cd ratios are listed in Table 1. The synthesis procedure of nanosized CdS was as follows: (a) to prepare a series of aqueous solutions including $\mathrm{CS}\left(\mathrm{NH}_{2}\right)_{2}$ and $\mathrm{Cd}\left(\mathrm{CH}_{3} \mathrm{COO}\right)_{2}$ according to their concentration listed in Table 1; (b) $50 \mathrm{~mL} \mathrm{CS}\left(\mathrm{NH}_{2}\right)_{2}$ solution was dropped into $50 \mathrm{~mL} \mathrm{Cd}\left(\mathrm{CH}_{3} \mathrm{COO}\right)_{2}$ solution to form transparent solution under stirring for $30 \mathrm{~min}$ and then heated at $100^{\circ} \mathrm{C}$ for $10 \mathrm{~h}$ so as to get a yellow single resource complex precursor of $\mathrm{Cd}\left(\mathrm{CS}\left(\mathrm{NH}_{2}\right)_{2}\right)_{2}\left(\mathrm{CH}_{3} \mathrm{COO}\right)_{2}$ (1) which will turn to $\mathrm{CdS}$ after calcination at $300^{\circ} \mathrm{C}$ for $0.5 \mathrm{~h}$. All other products were gaseous (2). Thus there are no any other byproducts in the thermolysis process. The final products were washed twice by ethanol and deionized water followed by drying at $100^{\circ} \mathrm{C}$ for $10 \mathrm{~h}$. The synthesized $\mathrm{CdS}$ samples are used after grinding to powder,

$$
\begin{aligned}
& \mathrm{Cd}\left(\mathrm{CH}_{3} \mathrm{COO}\right)_{2}+\mathrm{CS}\left(\mathrm{NH}_{2}\right)_{2} \\
& \quad \longrightarrow \mathrm{Cd}\left(\mathrm{CS}\left(\mathrm{NH}_{2}\right)_{2}\right)_{2}\left(\mathrm{CH}_{3} \mathrm{COO}\right)_{2} \\
& \mathrm{Cd}\left(\mathrm{CS}\left(\mathrm{NH}_{2}\right)_{2}\right)_{2}\left(\mathrm{CH}_{3} \mathrm{COO}\right)_{2} \longrightarrow \mathrm{CdS}+\mathrm{NH}_{3} \\
& \quad+\mathrm{HCNS}+\mathrm{CH}_{3} \mathrm{COCH}_{3}+\mathrm{CH}_{2} \mathrm{CO}
\end{aligned}
$$

2.2. Characterization of Photocatalysts. The powder X-ray diffraction (XRD) patterns were recorded on a Rigaku D/MAX-2500 diffractometer with $\mathrm{Cu} \mathrm{K}_{\alpha}(\lambda=1.5406 \AA)$ radiation. The transmission electron microscope (TEM) images were taken on a Hitachi800 electron microscope. The UV-vis diffuse reflectance spectra were measured on a Shimadzu UV-2550 spectrometer. The FT-IR spectra of the samples dispersed in $\mathrm{KBr}$ pellets were obtained within the $4000-400 \mathrm{~cm}^{-1}$ wavenumbers on a Nicolet $370 \mathrm{MCT}$ spectrometer.

2.3. Photocatalytic Testing. A 500-W Xe lamp was used as the light source and the visible wavelength was controlled through a 420-nm cut filter (LF420, China), which was hanged in a dark box and kept at about $15 \mathrm{~cm}$ above the liquid level. Aqueous suspensions of Rhodamine B (RB) dye $(100 \mathrm{~mL}$, with an initial concentration of $1.5 \times$ $\left.10^{-4} \mathrm{M}\right)$ and photocatalyst powder $(100 \mathrm{mg})$ were placed in a $250 \mathrm{~mL}$ beaker. Prior to irradiation, the suspensions were magnetically stirred for 10 min under dark condition to

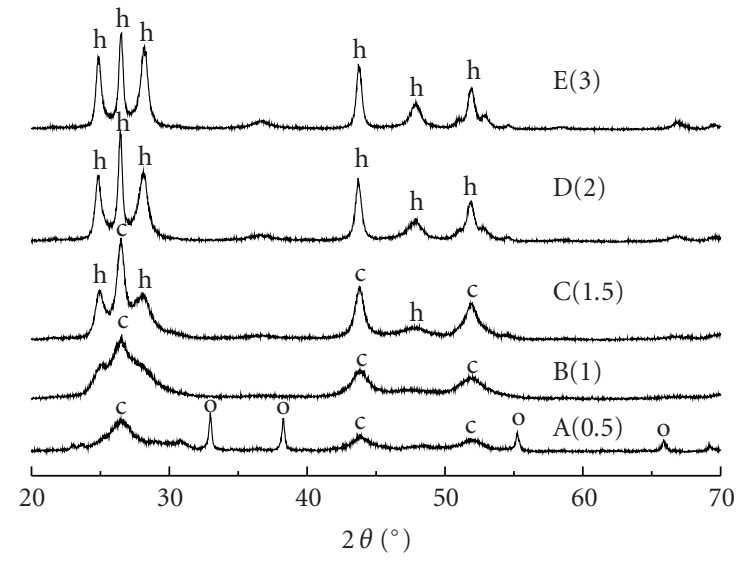

CdO: o

CdS: c-cubic h-hexagonal

FIgure 1: XRD patterns of CdS samples synthesized by different $n(\mathrm{~S}) / n(\mathrm{Cd})$ ratios of $\mathrm{A}: 0.5, \mathrm{~B}: 1, \mathrm{C}: 1.5, \mathrm{D}: 2$, and $\mathrm{E}: 3$.

establish an adsorption/desorption equilibrium between dye and photocatalyst surface. Under stirring, aliquots of a small amount of suspension (about $4 \mathrm{~mL}$ ) were taken out every 10 minutes under irradiation conditions, then centrifuged and analyzed using a SP-2000UV spectrophotometer (made in China).

\section{Results and Discussion}

3.1. XRD Characterization. XRD patterns of the CdS products are depicted in Figure 1. All the diffraction peaks of the nanocrystals are well indexed either as cubic CdS (JCPDF \# 65-2887) or as hexagonal CdS (JCPDF \# 77-2306) which have been marked by the symbol "c" or "h." When increasing thiourea/Cd molar ratio from 0.5 to $1,1.5,2$, and then to 3, a mixture of cubic $\mathrm{CdS}$ and $\mathrm{CdO}$ (Figure 1A), a cubic CdS (Figure 1B), a mixture of cubic and hexagonal CdS (Figure 1C), and hexagonal CdS (Figures 1D and 1E) were obtained, respectively.

The crystal phase of synthesized CdS changes regularly, from cubic phase $\rightarrow$ mixed phase $\rightarrow$ hexagonal phase. With the thiourea/Cd molar ratio of 0.5 , the excess cadmium acetate decomposes into $\mathrm{CdO}$ under heating condition. During the process of reaction, the cadmium acetate and thiourea molecules form a single source precursor of 


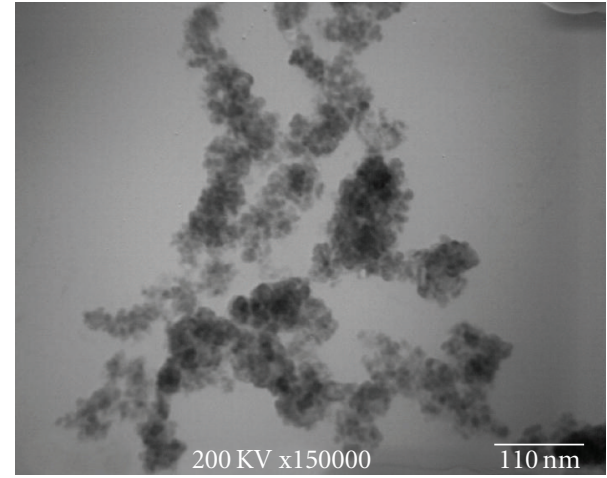

(a)

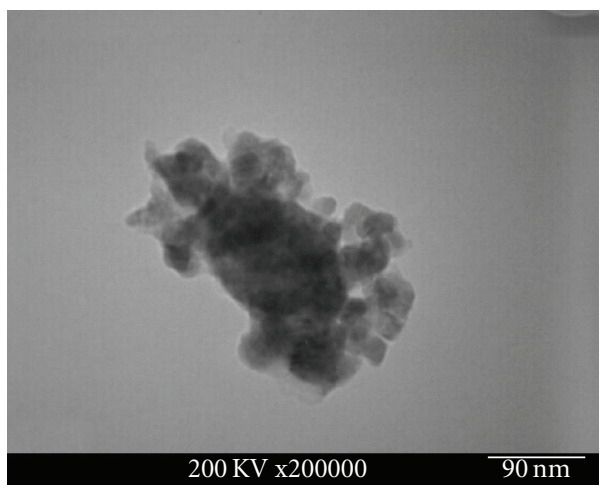

(c)

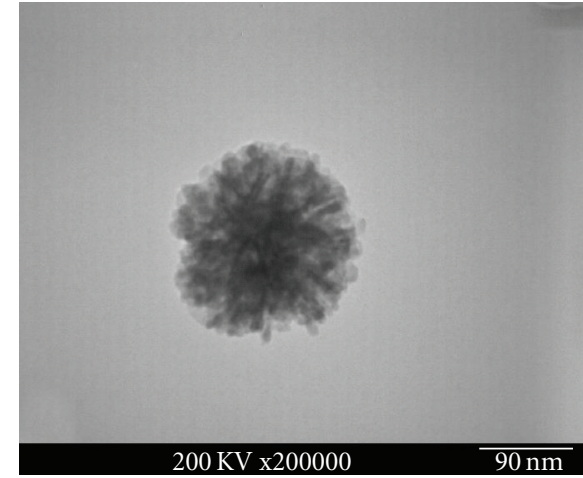

(b)

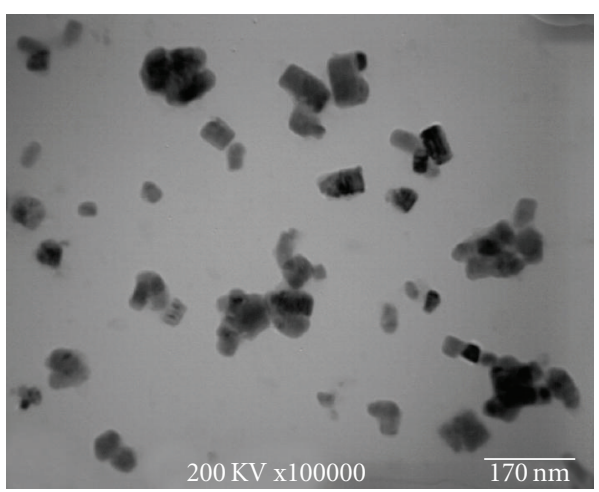

(d)

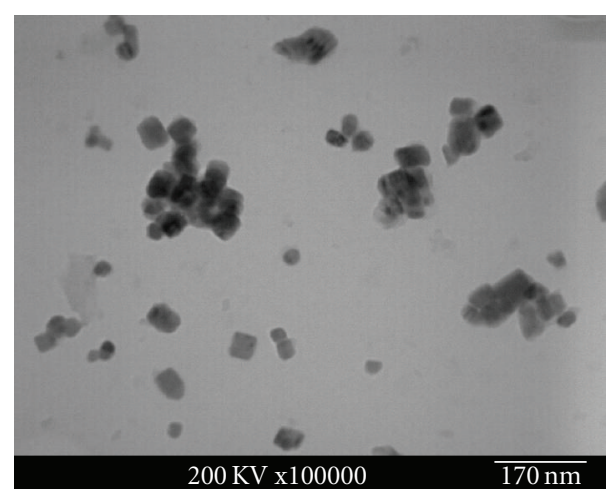

(e)

FIGURE 2: TEM images of CdS samples synthesized by different $n(\mathrm{~S}) / n(\mathrm{Cd})$ ratios of $\mathrm{A}: 0.5, \mathrm{~B}: 1, \mathrm{C}: 1.5, \mathrm{D}: 2$, and $\mathrm{E}: 3$.

Cd-thiourea complex $\mathrm{Cd}\left(\mathrm{SC}\left(\mathrm{NH}_{2}\right)_{2}\right)_{2}\left(\mathrm{CH}_{3} \mathrm{COO}\right)_{2}$, in which the $\mathrm{Cd}$ ion coordinates with four $\mathrm{S}$ atoms to form tetrahedral $\mathrm{CdS}_{4}$ block. In the cubic zinc blende structure, the pairs of interpenetrating tetrahedra are in the staggered conformation, while in the hexagonal wurtzite structure, these tetrahedra are in the eclipsed conformation. In the present study, for the Cd-thiourea- $\mathrm{CH}_{3} \mathrm{COO}$ - complex with a low thiourea/Cd ratio, the symmetry of the ligands is low, and there are steric hindrances to the formation of the $\mathrm{S}_{3} \mathrm{Cd}$ $\mathrm{SCd}_{3}$ group in the eclipsed conformation [9]. Thus, the lower symmetry of the first coordination sphere of $\mathrm{Cd}$ in a thiourea complex leads to a lower symmetry of the cubic CdS structure. In contrast, there are no such hindrances for the
Cd-thiourea- $\mathrm{CH}_{3} \mathrm{COO}$ - complex with a large thiourea/Cd ratio, and CdS crystallizes in the hexagonal wurtzite structure [10], which is thermodynamically more stable.

With increased S/Cd molar ratio, the grain size of CdS increases from $10 \mathrm{~nm}$ of the cubic phase (Figure 1A), to $26 \mathrm{~nm}$ of the hexagonal phase (Figure 1E), as shown in Table 1, that indicates that the change of grain size is an important factor affecting the phase transition of CdS [11] except for the symmetry change abovementioned.

3.2. TEM Characterization. The TEM images of CdS samples synthesized with different S/Cd ratios were shown in Figure 2. It is shown that the particles of sample A consisting 


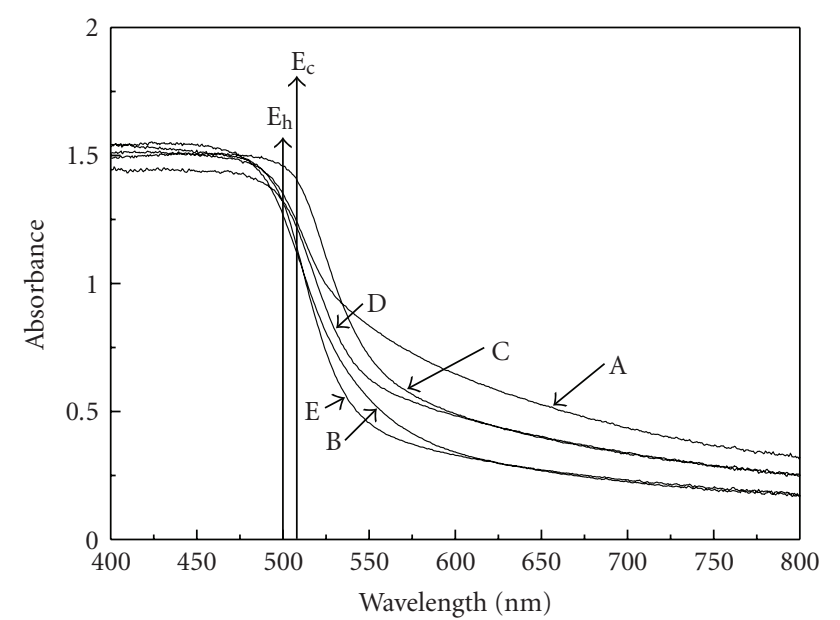

Figure 3: UV-vis spectra of CdS samples synthesized by different $n(\mathrm{~S}) / n(\mathrm{Cd})$ ratios of $\mathrm{A}: 0.5, \mathrm{~B}: 1, \mathrm{C}: 1.5, \mathrm{D}: 2$, and $\mathrm{E}: 3$.

of $\mathrm{CdO}$ and cubic CdS are about $10 \mathrm{~nm}$ exhibiting chain-like aggregates. The morphology of sample B seems as flocculent flower with diameter of about $100 \mathrm{~nm}$ which is consisting of much smaller particles and fiber aggregates. In the center of sample $\mathrm{C}$, a number of particles with the diameter of 10 to $20 \mathrm{~nm}$ are separated out corresponding to the mixed phases of cubic and hexagonal CdS, as shown in Figure 1C. With increased S/Cd molar ratio, the morphology of the samples becomes more perfect and homogeneous crystal of cubic CdS with diameter of about $20 \sim 30 \mathrm{~nm}$ that is a good agreement in the calculation results (Table 1), as shown in Figures 2(d) and 2(e). It is worth mentioning that the morphologies of CdS change subtly with a gradual increase of $\mathrm{S} / \mathrm{Cd}$ ratio, suggesting that the $\mathrm{S} / \mathrm{Cd}$ ratio plays a key role in the formation of diverse CdS nanostructures.

3.3. UV-Vis Characterization. All CdS samples synthesized with different $\mathrm{S} / \mathrm{Cd}$ ratios show strong light absorption at the wavelength of less than $550 \mathrm{~nm}$, and the consequent weak absorption even extends to $800 \mathrm{~nm}$, as shown in Figure 3 which indicates that the CdS samples have broad light absorption in the whole visible region. The absorbance has the order of: $\mathrm{A}>(\mathrm{C}, \mathrm{D})>(\mathrm{B}, \mathrm{E})$, in which samples $\mathrm{A}$ and $\mathrm{C}$ are the mixed phase of cubic $\mathrm{CdS}$ and $\mathrm{CdO}$, or of cubic and hexagonal CdS, respectively, while sample B is the pure cubic CdS, and samples D and $\mathrm{E}$ are pure hexagonal CdS. It is well known that the intrinsic energy gap of pure cubic or hexagonal CdS is $E_{\text {cubic }}=2.41 \mathrm{eV}$ or $E_{\text {hexagonal }}=2.47 \mathrm{eV}$ [12], corresponding to the cutoff absorption wavelength of $E_{\mathrm{c}}(\lambda=$ $515 \mathrm{~nm})$ or $E_{\mathrm{h}}(\lambda=502 \mathrm{~nm})$, respectively, as shown by the vertical lines in Figure 3. Thus all CdS samples synthesized with different S/Cd ratios undergo a redshift compared to the intrinsic adsorption of cubic or hexagonal CdS.

3.4. IR Characterization. The absorption bands of 3300 $3400 \mathrm{~cm}^{-1}$ and $1607-1645 \mathrm{~cm}^{-1}$ are assigned to the N$\mathrm{H}$ stretching and $-\mathrm{NH}_{2}$ bending vibrations of thiourea molecule [13], respectively, whose intensities gradually

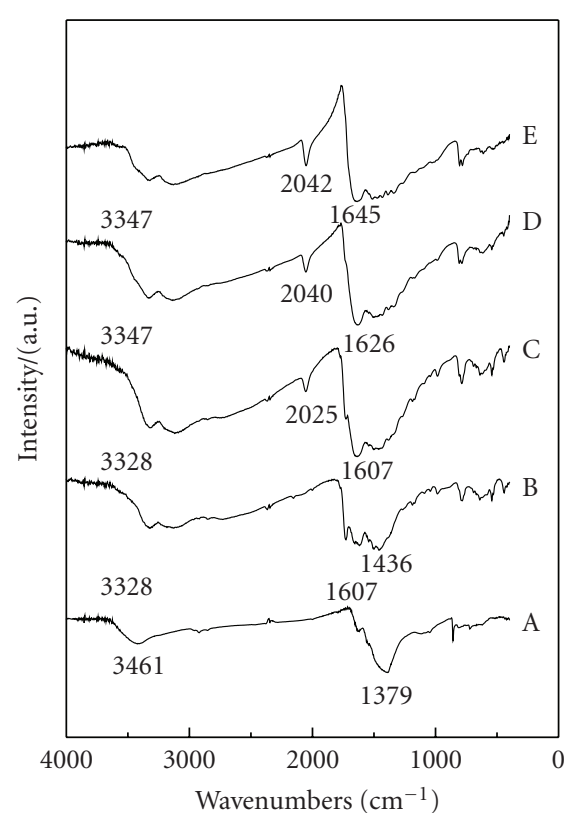

FIGURE 4: IR spectra of CdS samples synthesized by different $n(\mathrm{~S}) / n(\mathrm{Cd})$ ratios of $\mathrm{A}: 0.5, \mathrm{~B}: 1, \mathrm{C}: 1.5, \mathrm{D}: 2$, and $\mathrm{E}: 3$.

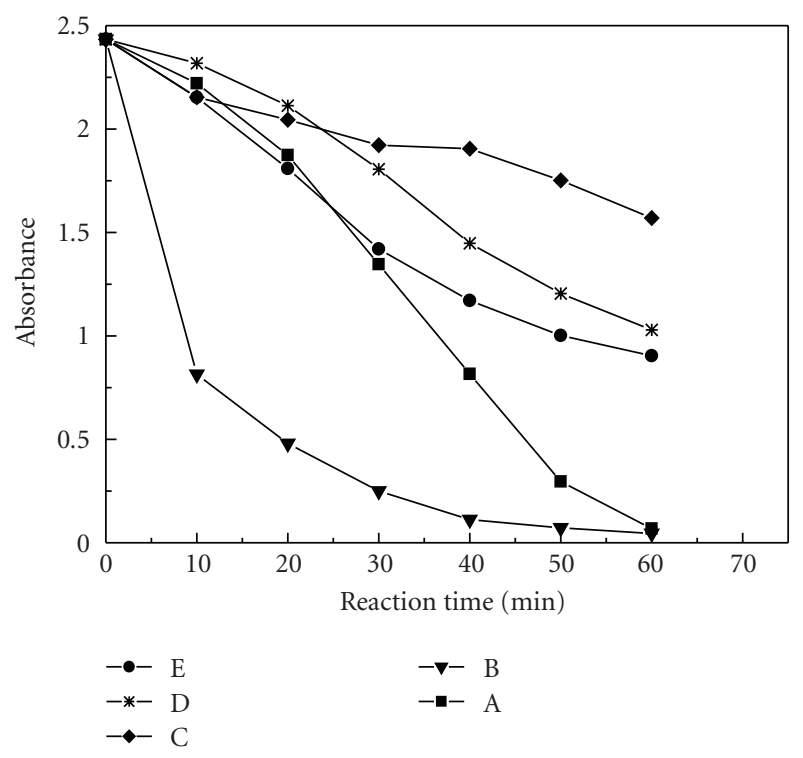

FIGURE 5: Photocatalytic degradation curves of RB using CdS samples synthesized by different $n(\mathrm{~S}) / n(\mathrm{Cd})$ ratios of $\mathrm{A}: 0.5, \mathrm{~B}: 1$, $\mathrm{C}: 1.5, \mathrm{D}: 2$, and $\mathrm{E}: 3$.

increase with increasing S/Cd ratios, as shown in Figure 4. Similarly, the absorption bands of $2040 \mathrm{~cm}^{-1}$ also gradually increase in samples of $\mathrm{C}, \mathrm{D}$, and $\mathrm{E}$, which is attributed to the infrared vibration of $-\mathrm{C} \equiv \mathrm{N}$ bond of thiocyanate (HCNS, H-S-C $\equiv \mathrm{N}$ ) produced in reaction (2). However, the characteristic diffraction peaks of thiocyanate or its derivatives do not appear in Figure 1, which indicates that the real content of decomposed products in the CdS sample is very low $(<1 \%)$. In contrast to the above situation, the IR absorption peaks near $2040 \mathrm{~cm}^{-1}$ of thiocyanate do not 
appear in the samples of $\mathrm{A}(0.5)$ and $\mathrm{B}(1.0)$, indicating that there is no excess thiourea to be decomposed into thiocyanate.

3.5. Photocatalytic Activities. The curves of photocatalytic degradation of RB using different $\mathrm{CdS}$ samples are shown in Figure 5. With the residual absorbance of RB after $60 \mathrm{~min}$ as evaluation index, the degradation activity is in the order of $\mathrm{B} \approx \mathrm{A}>E>D>C$. Sample $\mathrm{B}$ has relatively rapid degradation within the first $10 \mathrm{~min}$, which can be attributed to its strong adsorption of RB due to the flocculent particle morphology with larger surface area, as shown in Figure 2(b). Furthermore, sample A has the strongest absorption in visible region and the smallest grain size $(10 \mathrm{~nm})$, so it has a higher photocatalytic activity. While $n(\mathrm{~S}) / n(\mathrm{Cd})>1$, the catalytic activities of samples E, D, and $\mathrm{C}$ are lower than that of A and B. It is known from Figure 4 that the excess thiocyanate remains on the surface of samples $\mathrm{E}, \mathrm{D}$, and $\mathrm{C}$, in which the $\mathrm{S}^{2-}$ ions can act as a photoinduced hole sacrificial reagent and compete with the oxidation of $\mathrm{RB}$ dye, resulting in the decrease of the photocatalytic activity [14]. On the other hand, the order of phase composition effect on the catalytic activity is cubic > hexagonal > mixed. As shown in Figure 2, the hexagonal CdS samples of $\mathrm{D}$ and $\mathrm{E}$ are in good crystallinity and homogeneous particle dispersion. However, the mixed phase CdS of $\mathrm{C}$ is an aggregate containing many defects between the two-phase interface, accounting for the lower photocatalytic activity.

\section{Conclusions}

The cubic CdS, hexagonal CdS, and their mixed phase were synthesized under the conditions of thiourea/Cd $(\mathrm{S} / \mathrm{Cd})$ molar ratios $(0.5 \sim 3)$ of $\leq 1, \geq 2$, and $1.0 \sim 1.5$, respectively. The grain size of CdS increases with the increased S/Cd ratio. The photocatalytic activity is in order of cubic $>$ hexagonal $>$ mixed phase, in which the cubic phase CdS has the best photocatalytic activity for RB degradation due to its strong adsorption, intense absorbance in visible region, and the smaller grain size.

\section{Acknowledgments}

This work was financially supported by the Chinese National TCM Project in the 11th Five-Year Period through the Grant 2007BAK26B08. The authors acknowledge the Chinese People's Security University for its full cooperation.

\section{References}

[1] W. Kelsall, I. W. Hamley, and M. Geoghegan, Nanoscale Science and Technology, John Wiley \& Sons, New York, NY, USA, 2005.

[2] C. P. Poole and F. J. Owens, Introduction to Nanotechnology, John Wiley \& Sons, New York, NY, USA, 2003.

[3] D. Meissner, R. Memming, and B. Kastening, "Photoelectrochemistry of cadmium sulfide. 1. reanalysis of photocorrosion and flat-band potential," Journal of Physical Chemistry, vol. 92, no. 12, pp. 3476-3483, 1988.
[4] N. Sahu, M. K. Arora, S. N. Upadhyay, and A. S. K. Sinha, "Phase transformation and activity of cadmium sulfide photocatalysts for hydrogen production from water: role of adsorbed ammonia on cadmium sulfate precursor," Industrial and Engineering Chemistry Research, vol. 37, no. 12, pp. 46824688, 1998.

[5] G. Lin, J. Zheng, and R. Xu, "Template-free synthesis of uniform CdS hollow nanospheres and their photocatalytic activities," Journal of Physical Chemistry C, vol. 112, no. 19, pp. 7363-7370, 2008.

[6] N. Bao, L. Shen, T. Takata et al., "Facile Cd-thiourea complex thermolysis synthesis of phase-controlled CdS nanocrystals for photocatalytic hydrogen production under visible light," Journal of Physical Chemistry C, vol. 111, no. 47, pp. 1752717534, 2007.

[7] D. Jing and L. Guo, "A novel method for the preparation of a highly stable and active CdS photocatalyst with a special surface nanostructure," Journal of Physical Chemistry B, vol. 110, no. 23, pp. 11139-11145, 2006.

[8] J. Liu, W. Qin, S. Zuo, Y. Yu, and Z. Hao, "Solvothermalinduced phase transition and visible photocatalytic activity of nitrogen-doped titania," Journal of Hazardous Materials, vol. 163, no. 1, pp. 273-278, 2009.

[9] A. V. Naumov, V. N. Semenov, and E. G. Goncharov, "Properties of CdS films prepared from thiourea complexes of cadmium salts," Inorganic Materials, vol. 37, no. 6, pp. 539$543,2001$.

[10] V. Swayambunathan, D. Hayes, K. H. Schmidt, Y. X. Liao, and D. Meisel, "Thiol surface complexation on growing CdS clusters," Journal of the American Chemical Society, vol. 112, no. 10 , pp. 3831-3837, 1990.

[11] N. Herron, Y. Wang, and H. Eckert, "Synthesis and characterization of surface-capped, size-quantized CdS clusters. Chemical control of cluster size," Journal of the American Chemical Society, vol. 112, no. 4, pp. 1322-1326, 1990.

[12] D. J. Kim, Y.-M. Yu, J. W. Lee, and Y. D. Choi, "Investigation of energy band gap and optical properties of cubic CdS epilayers," Applied Surface Science, vol. 254, no. 22, pp. 75227526, 2008.

[13] G. W. C. Kaye and T. H. Laby, Tables of Physical and Chemical Constants, Longman Press, London, UK, 16th edition, 1995.

[14] I. Tsuji, H. Kato, and A. Kudo, "Photocatalytic hydrogen evolution on $\mathrm{ZnS}-\mathrm{CuInS}_{2}-\mathrm{Ag} \ln \mathrm{S}_{2}$ solid solution photocatalysts with wide visible light absorption bands," Chemistry of Materials, vol. 18, no. 7, pp. 1969-1975, 2006. 


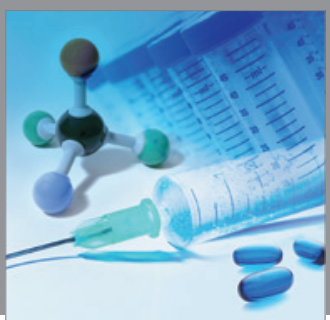

International Journal of

Medicinal Chemistry

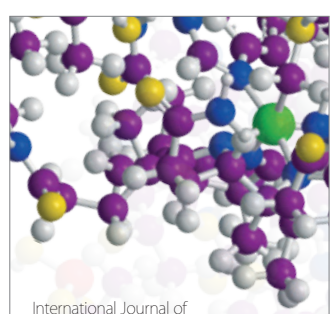

Carbohydrate Chemistry

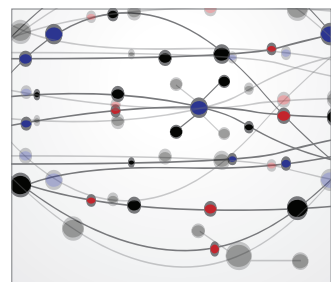

The Scientific World Journal
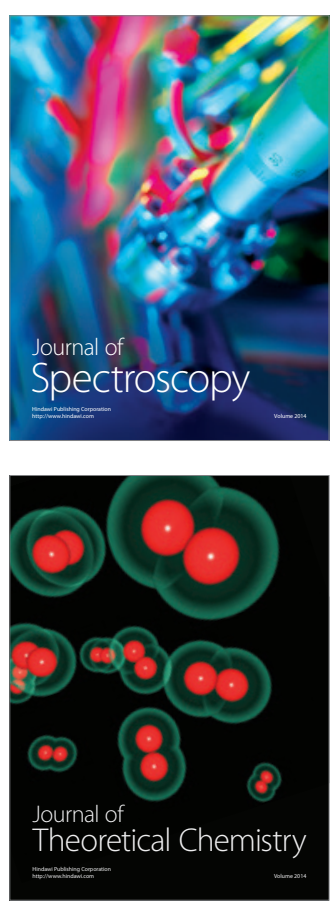
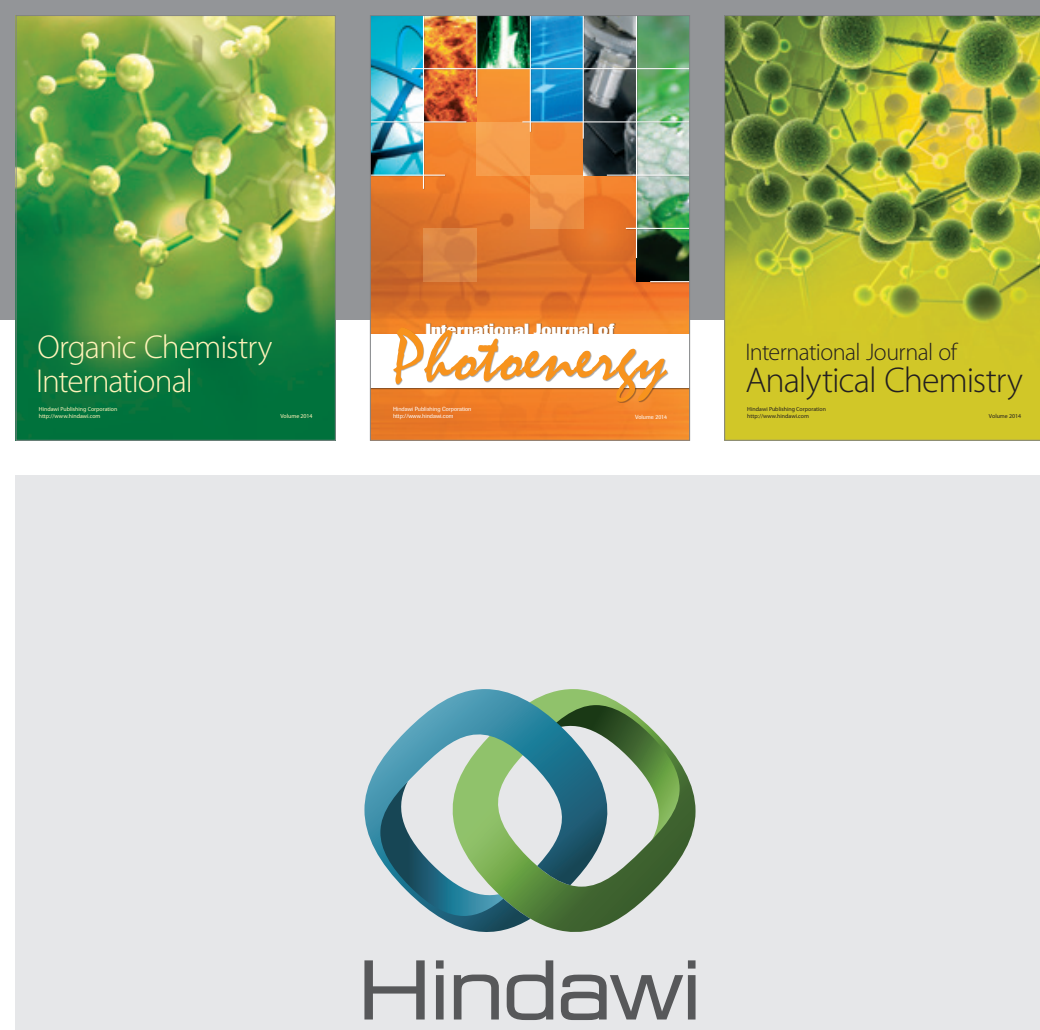

Submit your manuscripts at

http://www.hindawi.com
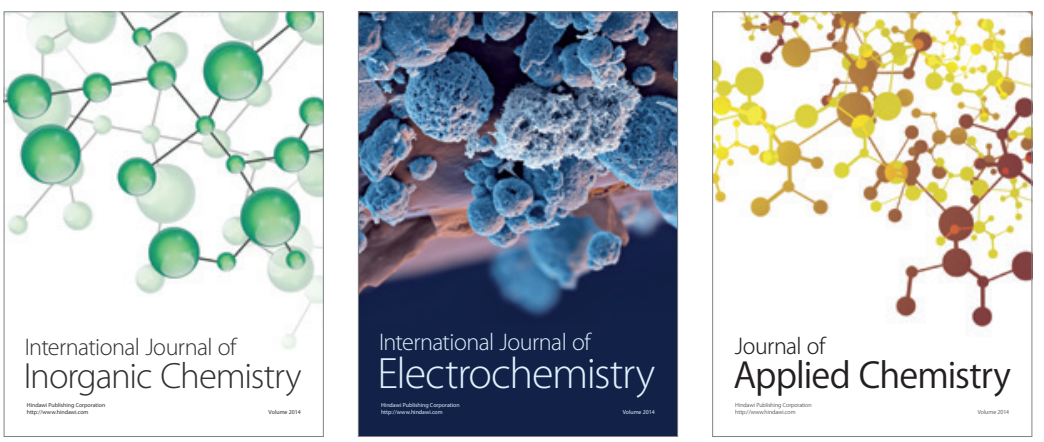

Journal of

Applied Chemistry
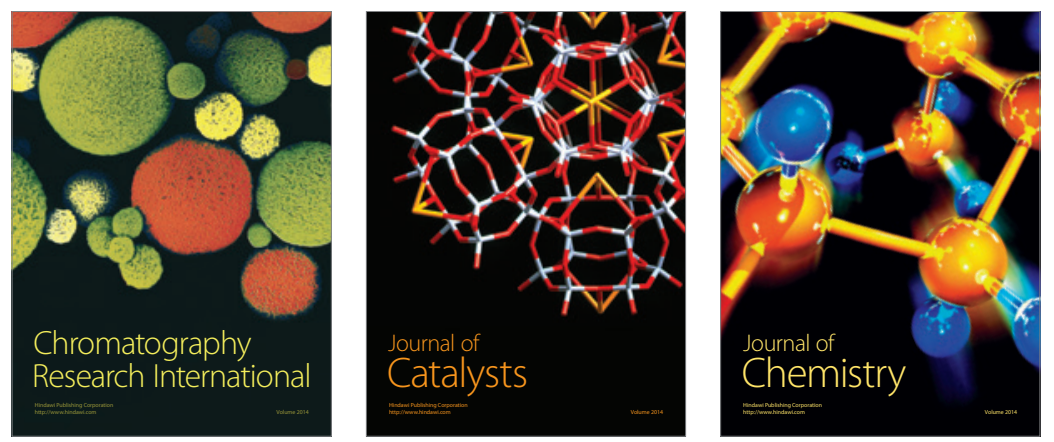
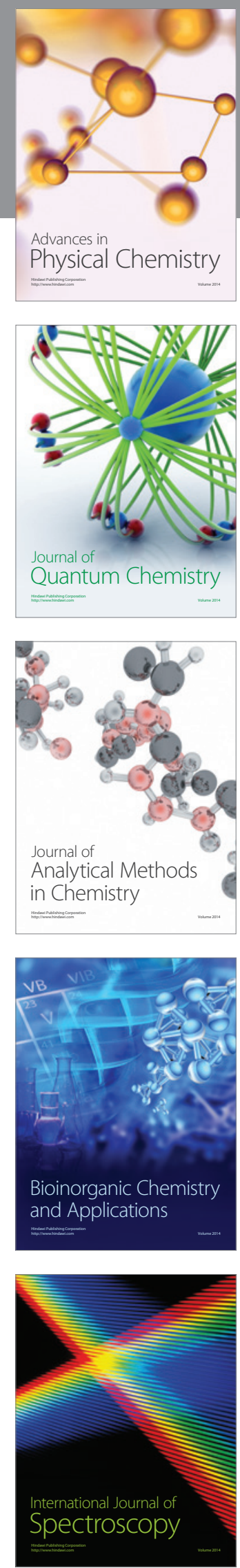